\title{
Ultra-Short-Term Prediction Intervals of Photovoltaic AC Active Power
}

\author{
E. Scolari, D. Torregrossa, J.-Y. Le Boudec, M. Paolone \\ École Polytechnique Fédérale de Lausanne (EPFL) \\ Lausanne, Switzerland
}

\begin{abstract}
The paper describes a heuristic method for the ultra-short-term computation of prediction intervals (PIs) for photovoltaic (PV) power generation. The method allows for directly forecasting the AC active power output of a PV system by simply extracting information from past time series. Two main approaches are investigated. The former relies on experimentally observed correlations between the time derivative of the PV $\mathrm{AC}$ active power output and the errors caused by a generic point forecast technique. The latter approach represents an improvement of the first one, where the mentioned correlations are clustered as a function of the value of the $\mathrm{AC}$ active power.

The work is framed in the context of microgrids and inertialess power systems control, where accounting for the fastest dynamics of the solar irradiance can become extremely valuable. We validate the proposed model using one month of $\mathrm{AC}$ active power measurements and for sub-second time horizons: 100, 250 and $500 \mathrm{~ms}$.
\end{abstract}

Keywords-forecast, photovoltaic, prediction intervals, short-term

\section{INTRODUCTION}

Today's increasing connection of distributed energy resources (DERs) in low and medium voltage power systems poses severe limitations in the operation of electrical distribution grids. Currently, one of the major efforts of the power system community is the definition of optimal control methodologies able to account for the high volatility of renewable DERs. The accurate forecasting of stochastic resources is particularly important for their massive integration into the grid and for the development of associated robust control techniques. In this context, the selection of the forecast time horizon depends on the needs associated with the real-time control of DERs to ensure a reliable operation of the grid.

In this paper, we focus on the $\mathrm{AC}$ active power forecast of photovoltaic (PV) generation with a sub-second time resolution. Indeed, for power distribution systems integrating PV panels, the volatility of the solar irradiance in time scales below a second can largely impact the grid operation, [1]. These dynamics can involve high value of the time derivative of the injected AC active power if the PV panels are coupled with inverters equipped with a Maximum Power Point Tracking (MPPT) control capable to react with time scales similar to the solar irradiance variations, e.g. [2]. When referring to low inertia microgrids in which loads, storage and generation are interfaced with the grid via power electronics, the requirements of the forecasting tools are different from those associated to large scale power transmission grids. Indeed, it becomes very valuable to quantify the fastest volatility of DERs, since these variations might impact the local grid security of supply. An example is represented by the microgrid islanding maneuver that might require forecast information at sub-second time scale to correctly adjust the gains of the slack resource droop control.

The work aims at implementing a method to compute prediction intervals (PIs) for PV AC active power. Probabilistic forecast is important in microgrids robust control as it represents a fundamental information for decision making under uncertainty, besides the more common single-point forecast [3]. The computed PIs target a sub-second time horizon from $100 \mathrm{~ms}$ up to $500 \mathrm{~ms}$ (i.e., ultra-short-term). The proposed method, called Dynamic Interval Predictor (DIP), was firstly introduced in [1] for sub-second irradiance forecast and it is here improved and extended in several aspects. First, the methodology is applied to directly forecast the PV AC active power by only using past AC power measurements and without the need of any irradiance sensing system. The direct forecast of the AC active power has the advantage of avoiding the introduction of an intermediate model of the PV system. The DIP is based on the experimentally verified correlation between the derivative of the PV AC active power and the errors caused by a generic point forecast method. Differently from [1], we compute here the absolute error instead of the relative one since it proved to be more indicative of the forecast uncertainty, as explained in subsection IV-B. Moreover, we propose an improved approach that consists in clustering the mentioned correlations as a function of the AC active power value itself. In other words, we consider the value of the $\mathrm{AC}$ active power as a further variable that is expected to influence the error caused by the point forecast. As a further last step beyond [1], we describe how the algorithm can be embedded into an industrial microcontroller and adopted within a realtime control framework. As example, we refer to the control solution introduced in [4], [5], where software agents, specific for each resource, make use of the PIs to compute deviceagnostic belief functions. In this applications the agents are able to communicate among each other's using a simple protocol with a refresh rate of around $100 \mathrm{~ms}$ (Section II).

The paper is structured as follows. Section II introduces the COMMELEC control framework. Section III describes the state of the art for short-term forecasting. Section IV illustrates the experimental setup and shows the correlations basis for the proposed methodologies. Section V describes the structure of the method, considering two different implementations. 
Section VI analyses the performance of the DIP and compare it with benchmark methods. Furthermore, an overview of the deployment of the DIP into an industrial hardware platform is given. In the last section conclusions are presented.

\section{THE COMMELEC FRAMEWORK}

The proposed DIP is used in the context of the COMMELEC control framework described in [4], [5]. This framework has been introduced for real-time control of the electrical grid. It is based on the idea that resources can directly communicate to define explicit set-points of active and reactive power in a sub-second time scale. Software Agents (deployed in dedicated microcontrollers) are responsible for resources (loads, storage systems, PVs, etc.), and communicate by using an advertise/request protocol. In particular, a Grid Agent (GA) is responsible for the quality of service in a portion of a distribution network. The GA receives advertisements from each Resource Agent (RA) that specifies the follower capabilities and expected behavior. It is important to notice that the GA can itself be a follower of a higher level agent from which it can receive a requested power set-point. The GA has to compute the requested set-points to send to the RAs and to aggregate information to send to its own leader by solving an optimization problem.

The main task of a RA (e.g., the PV one) is to translate its specifics into the abstract framework which consists of three main elements: PQ profile, Virtual Cost (VC) and Belief Function (BF). The PQ profile represents the region (of active and reactive power) where the subsystem can inject or absorb power. The VC quantifies the propensity of a system to stay in a particular zone of the PQ profile. The BF accounts for the uncertainty of the subsystem operation: it returns the set of all possible set-points that the system might implement due to its own stochasticity.

PVs are among the most volatile resources and their power generation profiles are mainly based on forecasts. Thus, quantifying the uncertainty of the forecast tool for short time horizons can be extremely valuable for real-time controllers. In our framework, we consider a PV RA that cannot be controlled and that is able to abstract its state only by means of the BF (i.e. it is a mere source of uncertainty). Fig. 1 shows the PQ profile and the BF of the uncontrollable PV agent (UPVA). The PQ profile is a singleton computed as the point forecast for the next time step while the BF is the computed PI (dashed lines). In our specific case the converter tracks a power factor of one (small variation on $\mathrm{Q}$ ).

\section{State Of the ART: PV Forecasting AND PREDICTION INTERVALS}

With exception of [1], the available literature mainly considers intra-day and intra-hour time horizons for irradiance and power forecast, while there is a lack of forecasting methods targeting time scales of seconds or sub-seconds.

Regarding methods for point prediction, most of the available literature focuses on solar irradiance forecast. In this context, different techniques can be classified based on the

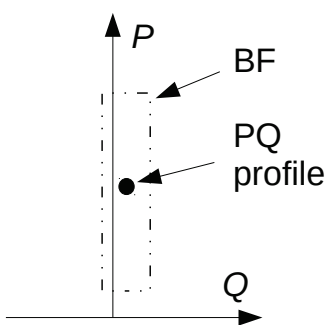

Fig. 1. Simplified representation of the advertizement sent by an UPVA.

target time horizon and space resolution, see [6], [7]. Forecasting techniques such as Numerical Weather Prediction (NWP) are used for time horizons ranging from several hours up to some days and for regional forecast. However, the limited spatial and temporal resolution makes them ineffective for intra-minutes/intra-hour time horizons. In general, the available methods for intra-hour forecast combine stochastic and artificial intelligence techniques, based on time series models. Examples include the usage of Artificial Neural Networks (ANNs), e.g. [8], and Auto-Regressive Integrated Moving Average (ARIMA) model, e.g [9]. Further methods propose the inclusion of local ground imaging. For example, images from a ground-based sky imager are used to detect cloud positions and motion on time horizons from $30 \mathrm{~s}$ up to 5 minutes, [10]. Authors of [11] use Total Sky Imagers (TSIs) to forecast the Direct Normal Irradiance (DNI) at the ground level for time horizons varying from 3 to $15 \mathrm{~min}$.

In general, irradiance forecasting implies the necessity of an intermediate model of the PV system and converter to compute the total power output. On the contrary, in the present work we are interested in directly forecasting the AC active power. In the context of power forecast, Authors of [12] developed several forecasting methods (Persistent model, ANNs, ARIMA, $\mathrm{k}$-Nearest-Neighbors) for predicting the power output of 1 and 2 hours ahead. Authors of [13] propose a method where first a normalization of the solar power is obtained using a clear sky model and then the normalized solar power is predicted using adaptive linear time series models.

Regarding the models for PIs computation, there is a general dependence of these models on the specific point forecast technique. In [14] the k-Nearest-Neighbors algorithm is used to predict the irradiance and to calculate PIs in the range of 5-30 minutes. Sky images are also included to improve the computation of the PIs. The method shows good results for the PIs computation of global horizontal irradiance, with high probability coverage $(\sim 90 \%)$ and narrow average normalized width $(\sim 8 \%)$. A hybrid, real-time solar forecasting model is shown in [15] to construct PIs of direct normal irradiance for intra-hour forecast horizons. The hybrid model uses images combined with support vector machine and ANN. Examples of PIs computation methods that are independent of the point forecast technique are shown in [16] and [17] in the context of wind power generation.

Assume to be in a generic time step $t_{k}$ in which we 
have available the PI estimations and the realized power. In accordance with its definition, (see [18]), a PI has to satisfy the following equation with a certain confidence level $\eta$ :

$$
\hat{P}_{P O W}^{k}-L_{P O W}^{k} \leq P_{P O W}^{k} \leq \hat{P}_{P O W}^{k}+U_{P O W}^{k} .
$$

with,

- $\hat{P}_{P O W}^{k}$ : predicted AC power output at time step $k$;

- $P_{P O W}^{k}$ : measured AC power output at time step $k$;

- $L_{P O W}^{k}$ : lower-bound of the prediction interval at time step $k$;

- $U_{P O W}^{k}$ : upper-bound of the prediction interval at time step $k$.

\section{EXPERIMENTAL EVIDENCES OF AC POWER VARIATION}

In this section we use a dedicated measurement setup to investigate the experimental correlation between the PV AC active power, its derivative and the errors caused by a given point forecast method. In this respect, we underline that the PV power fluctuation strongly depends on the variation of the solar irradiance and on the action of the MPPT controller adopted by the PV converter.

\section{A. Experimental Setup}

The experimental setup used for the validation of the proposed methodology is located at the following GPS coordinates: 46.518397-N, 6.565229-E. The PV plant consists of $255 \mathrm{~W}$ Polycrystalline modules connected to a $10 \mathrm{~kW}$ solar converter as follows: 2 branches with 14 modules each and one branch with 11 modules. In each branch the modules are connected in series. The DC/AC three-phase converter is equipped with two DC/DC converters each one implementing a separate MPPT. The AC voltages and currents at the converter AC output are measured and the active power is then computed by extracting the single tone information. Three LEM CV 3-100 Voltage Transducers are used to measure the voltage while three LEM LF 205-S Current Transducers are used as current sensors. The output of these sensors is sampled at $50 \mathrm{kHz}$ and the phasor measurements are then used to estimate the PV output power with a pace of $50 \mathrm{~ms}$.

Three different down-sampling times are considered to generate series of the PV AC active power : $100 \mathrm{~ms}, 250$ $\mathrm{ms}$ and $500 \mathrm{~ms}$. For the obtained time series, instantaneous values are used instead of average ones.

In what follows data collected between August and September 2015 are assigned as a validation set, considering a total period of one month. Only hours with daylight are included in the evaluation. The whole time series is shown in Fig. 2.

\section{B. Experimentally Observed Power Fluctuations}

As mentioned in Section I, significant AC active power fluctuations are observed at sub-second time scale for our PV installation. In order to provide an example, Fig. 3 shows the

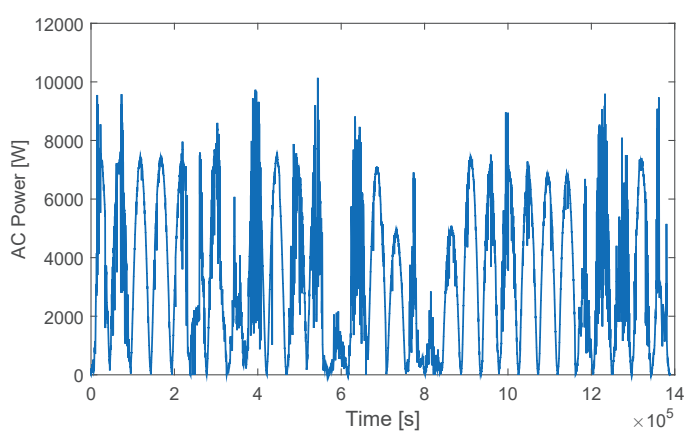

Fig. 2. PV AC active power measured between August and September 2015 (30 days). The plant is located at GPS coordinates 46.518397-N, 6.565229-E.

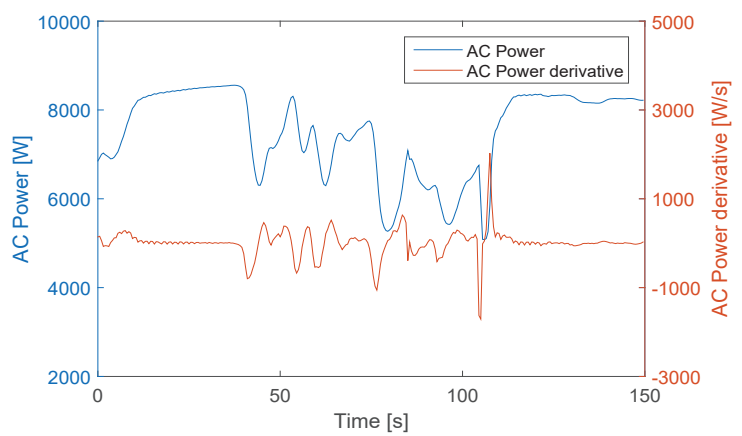

Fig. 3. Example of measured PV variations: AC active power and its time derivative. Time derivatives are calculated for a time horizon of $500 \mathrm{~ms}$.

time evolution of the $\mathrm{AC}$ active power along with its time derivative for a time window of $150 \mathrm{~s}$, considering a period of high solar variability. For our installation, the observed fluctuations can reach time derivatives of $2 \mathrm{~kW} / \mathrm{s}$. Considering that our PV plant has a rated power of $10 \mathrm{~kW}$, this represents a fluctuation of $20 \%$ of the PV capacity in about one second. As mentioned in the introduction, the DIP is based on the investigation of the correlation between the error of the point forecast computation $e_{P O W}^{k+1}$ at $t_{k+1}$ and the derivative of $\mathrm{AC}$ power $\dot{P}_{P O W}^{k}$ at $t_{k}$. Note that, differently from [1], $e_{P O W}^{k+1}$ is defined as the absolute error:

$$
e_{P O W}^{k+1}=P_{P O W}^{k+1}-\hat{P}_{P O W}^{k+1} .
$$

We noticed that using the relative error, defined as $e_{P O W}^{k+1} / P_{P O W}^{k+1}$, can be misleading. Indeed, high values of the relative error can be generated by low $\mathrm{AC}$ power values rather than by high power fluctuations. This means that we might have significant errors even if the $\mathrm{AC}$ power derivative is negligible. The improvement obtained by using the absolute error instead of the relative one is shown in Section VI-B.

The discrete time derivative is calculated as:

$$
\dot{P}_{P O W}^{k}=\frac{P_{P O W}^{k}-P_{P O W}^{k-1}}{\Delta t},
$$

where $\Delta t$ is the considered time step. 
Fig. 4 illustrates the 2.5\%-97.5\% quantile intervals of the absolute error (computed at $t_{k+1}$ ) associated to different ranges of the power derivative (computed at $t_{k}$ ). In this example, a time horizon of $250 \mathrm{~ms}$ is selected. For the sake of simplicity, the figure shows the absolute value of the power derivative (indeed, a symmetric behavior for negative and positive derivatives was observed). It is worth noticing that different quantiles of the error are associated to different power derivative ranges. Consequently, we are interested in investigating the possibility to statistically quantify the error made by the point forecast computation (at $t_{k+1}$ ) as a function of the $\mathrm{AC}$ power time derivative (at $t_{k}$ ). This consideration is the starting point of the DIP method, presented in the next section (Algorithm I).

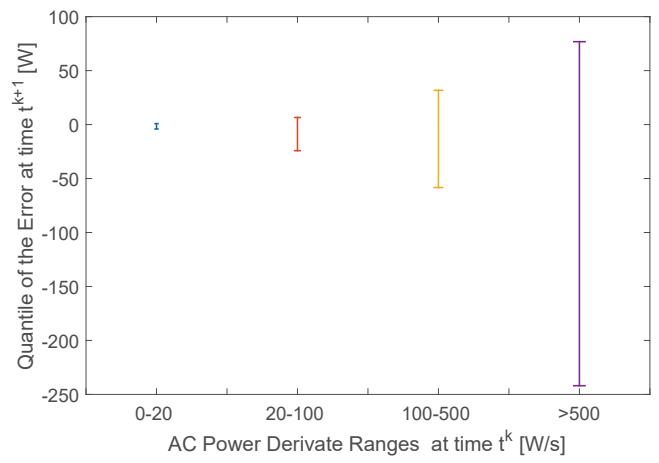

Fig. 4. 2.5\%-97.5\% quantile intervals of the error caused by the point forecast at $t_{k+1}$ for different ranges of the $\mathrm{AC}$ power time derivative at $t_{k}$.

As mentioned, we propose here an improved version of the DIP that considers the value of the AC active power as a further explanatory variable. The method is presented in the next section (Algorithm II). In this context, Fig. 5

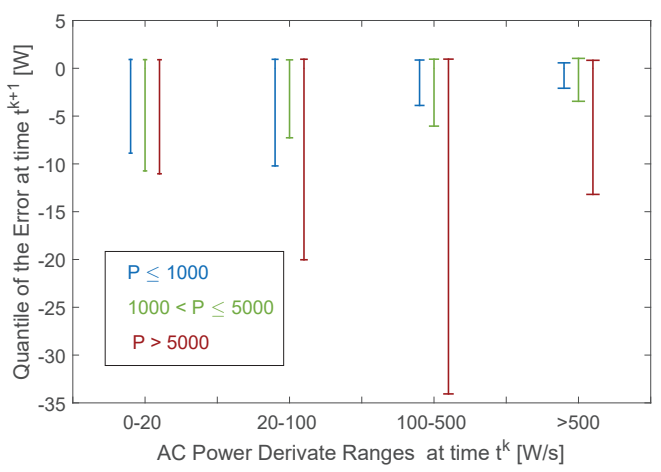

Fig. 5. 2.5\%-97.5\% quantile intervals of the error caused by the point forecast at $t_{k+1}$ for different ranges of the $\mathrm{AC}$ power time derivative at $t_{k}$. Measurements are clustered in three groups based on the AC active power.

illustrates the $2.5 \%-97.5 \%$ quantile intervals of the absolute error associated to different ranges of the power derivative, considering different ranges of the $\mathrm{AC}$ active power value (for clearness we show here only three ranges of $\mathrm{AC}$ active power, highlighted with different colors). Data are first clustered based on the power value and then, for each group, the quantiles of the error are calculated for different ranges of the power derivative. We can observe that, for a given derivative range, the error distribution depends on the considered power range, this suggesting that a further clustering in this direction can improve the performance. This is the main assumption behind the development of Algorithm II.

\section{Methodology}

\section{A. Dynamic Interval Predictor: Algorithm I}

In this first part we summarize the procedure introduced in [1] for computing the PIs.

The main idea of the DIP is to use the correlations defined by the conditional distribution matrix $\mathbf{R}$ to compute the PIs. We define $e(n)$ as the $n^{t h}$ discretized value of errors and $\dot{p}(m)$ as the $m^{t h}$ discretized value of derivatives. Then, $R(n, m)$ corresponds to the probability that the next forecast error is $e(n)$, given that the derivative of the $\mathrm{AC}$ active power is $\dot{p}(m)$.

The process to compute the PI is the following:

1) Define the nominal confidence level $\eta$;

2) At the generic time step $t_{k}$, calculate $\dot{P}_{P O W}^{k}$ as described in Eq. (3);

3) Find column $m$ for which the discretized value $\dot{p}(m)$ is the closest to $\dot{P}_{P O W}^{k}$;

4) Column $m$ of matrix $\mathbf{R}$ is considered as the histogram of a probability distribution that has a discrete cumulative distribution function $F()$. The matrix $\mathbf{R}$ returns the values of $F()$ at the points $e(n)$ as:

$$
F(e(n))=\sum_{n^{\prime}=1}^{n} R\left(n^{\prime}, m\right)
$$

5) Compute the upper and lower quantiles of the distribution of errors corresponding to column $m$ of matrix $\mathbf{R}$, associated to the confidence level $\eta$. The values $e_{P O W}^{U P P}$ and $e_{P O W}^{L O W}$ are obtained by the conditions:

$$
\begin{aligned}
& F\left(e_{P O W}^{L O W}\right)=\frac{1-\eta}{2}, \\
& F\left(e_{P O W}^{U P P}\right)=\frac{1+\eta}{2} .
\end{aligned}
$$

6) The PI widths are then obtained by:

$$
\begin{aligned}
& L_{P O W}^{k+1}=e_{P O W}^{L O W} \\
& U_{P O W}^{k+1}=e_{P O W}^{U P P} .
\end{aligned}
$$

The PI computation is based on the knowledge of the conditional distribution matrix $\mathbf{R}$. The matrix $\mathbf{R}$ is updated at each time step and it is fed by the raw sampled data of the PV AC active power.

The proposed update method is based on the following. Let assume that, at time $t_{k}$, we have a new observation, and let $e\left(n_{0}\right)$ and $\dot{p}\left(m_{0}\right)$ be the closest discretized values to the error $e_{P O W}^{k+1}$ and to the derivative $\dot{P}_{P O W}^{k}$, respectively. The update equations for matrix $\mathbf{R}^{N E W}$ are: 


$$
\begin{aligned}
R^{N E W}\left(n_{0}, m_{0}\right) & =\frac{N^{O L D}\left(n_{0}, m_{0}\right)+1}{N^{O L D}\left(m_{0}\right)+1}, \\
R^{N E W}\left(n, m_{0}\right) & =\frac{N^{O L D}\left(n, m_{0}\right)}{N^{O L D}\left(m_{0}\right)+1}, n \neq n_{0}, \\
R^{N E W}(n, m) & =R^{O L D}(n, m), n \neq n_{0}, m \neq m_{0},
\end{aligned}
$$

with:

- $N^{O L D}\left(m_{0}\right)$ is the number of observations having a derivative in the range of $\dot{p}\left(m_{0}\right)$;

- $N^{O L D}\left(n_{0}, m_{0}\right)$ is the number of observations having a an error in the range of $e\left(n_{0}\right)$ and a derivative in the range of $\dot{p}\left(m_{0}\right)$.

The superscripts $N E W$ and $O L D$ refer to the updated and the old version of the $\mathbf{R}$ matrix and the $\mathrm{N}$ variables, respectively.

\section{B. Dynamic Interval Predictor: Algorithm II}

In Algorithm II we add a third dimension to matrix R. In particular we introduce $p(l)$ as the $l^{\text {th }}$ discretized value of AC active powers. Then, $R(n, m, l)$ corresponds to the probability that the next forecast error is $e(n)$, given that the derivative of the irradiance is in the range of $\dot{p}(m)$ and the measured $\mathrm{AC}$ active power is in the range of $p(l)$. Then, the process to compute the PIs is:

1) Define the nominal confidence level $\eta$;

2) Find the layer $l$ for which the discretized power value $p(l)$ is the closest to our AC power measurement $P_{P O W}^{k}$.

3) Then, the procedure corresponds to the one described for Algorithm I (numbers 2-6), but applied to the the selected layer $l$ of matrix $\mathbf{R}$.

We assume that, at time $t_{k}$, we have a new observation and $p\left(l_{0}\right)$ is the closest discretized AC power value to the measurement $P_{P O W}^{k}$. Then, Let $e\left(n_{0}\right)$ and $\dot{p}\left(m_{0}\right)$ be the closest discretized values to the error $e_{P O W}^{k+1}$ and to the derivative $\dot{P}_{P O W}^{k}$, respectively. The update equations for matrix $\mathbf{R}^{N E W}$ are:

$$
\begin{aligned}
R^{N E W}\left(n_{0}, m_{0}, l_{0}\right) & =\frac{N^{O L D}\left(n_{0}, m_{0}, l_{0}\right)+1}{N^{O L D}\left(m_{0}, l_{0}\right)+1}, \\
R^{N E W}\left(n, m_{0}, l_{0}\right) & =\frac{N^{O L D}\left(n, m_{0}, l_{0}\right)}{N^{O L D}\left(m_{0}, l_{0}\right)+1}, n \neq n_{0}, \\
R^{N E W}(n, m, l) & =R^{O L D}(n, m, l), n \neq n_{0}, m \neq m_{0}, l \neq l_{0} .
\end{aligned}
$$

\section{RESUlts AND DisCUSSION}

First, we show the improvement obtained by using the absolute error definition instead of the relative one to deliver our PIs. Then, the DIP is applied to three different point forecast techniques to prove that the algorithm can be coupled with different point forecast methods while maintaining good performance. Additionally, the proposed DIP is compared with two benchmark methods used to calculate the PIs. To ensure a fair comparison, the Holt Winter (HW) method is selected as point forecaster for this second analysis. The HW is based on exponential smoothing, it analyzes seasonal time series directly and has a simple model formulation, [19]. These first results are all obtained by implementing Algorithm I but analogue conclusions can be drawn when applying Algorithm II. Then, a further analysis is carried out to compare the two proposed algorithms (I and II). For the testing results shown hereafter, PIs are constructed at a nominal confidence level of $95 \%$. The evaluation of the performance is done by using power measurements covering the period of 30 days shown in Fig. 2.

In order to analyze our results, it is necessary to define specific performance metrics.

\section{A. Metrics}

According to the available literature regarding PIs performance evaluation, e.g. [15], [20], we implement three metrics to quantify the performance of the proposed method. The first one is the PI coverage probability (PICP) which accounts for the number of times that the realization is inside the PI for a given nominal confidence level $\eta$, namely:

$$
P I C P=\frac{1}{M} \sum_{k=1}^{M} c_{k},
$$

where $c_{k}=1$ if the realization is inside the PI, $c_{k}=0$ otherwise. $M$ is the total number of forecast instances.

Then, to account for the fact that the wider the PI, the easier it is to have a realization falling inside it, we compute the PI normalized averaged width (PINAW):

$$
P I N A W=\frac{1}{M P_{\max }} \sum_{k=1}^{M}\left(U_{P O W}^{k}-L_{P O W}^{k}\right) .
$$

In this case $P_{\max }$ is equal to $10 \mathrm{~kW}$. The last metric quantifies the trade-off between having a good coverage probability and low interval width and it is called coverage width-based criterion (CWC).

$$
C W C=P I N A W\left(1+\gamma(P I C P) e^{-\alpha(P I C P-\eta)}\right),
$$

where

$$
\gamma= \begin{cases}0, & \mathrm{PICP} \geq \eta \\ 1, & \mathrm{PICP}<\eta\end{cases}
$$

$\alpha$ is a parameter that can be tuned between 50 and 100 based on how much we want to penalize bad PIs, see [20]. We fix this value to 50 .

\section{B. Error Definition}

Table I shows the performance improvement obtained by using the absolute error instead of the relative one to build our algorithms and compute the PIs. Results are shown for Algorithm I but an equivalent improvement was found for Algorithm II (not shown here to avoid redundancy). Different columns refer to different time horizons of 100, 250 and 500 $\mathrm{ms}$, respectively. We can see that, when using the relative error, the PICP is below the nominal confidence level even with 
TABLE I

PERFORMANCE COMPARISON: RELATIVE AND ABSOLUTE ERROR.

\begin{tabular}{|c|c|c|c|}
\hline \multicolumn{4}{|c|}{ PICP-PINAW-CWC \% } \\
\hline Time Horizon & $100 \mathrm{~ms}$ & $250 \mathrm{~ms}$ & $500 \mathrm{~ms}$ \\
\hline Abs. Error & $95.15-0.059-0.059$ & $95.47-0.12-0.12$ & $95.44-0.27-0.27$ \\
\hline Rel. Error & $94.68-0.38-0.79$ & $94.75-0.55-1.13$ & $94.70-0.72-1.15$ \\
\hline
\end{tabular}

a higher value of PINAW. This holds for all the considered forecast horizons.

All the results shown hereafter are obtained considering the absolute error distribution to build the matrix $\mathbf{R}$ and deliver our PIs.

\section{Point forecast Method Independence}

In this section we show that the proposed DIP can be coupled with generic point forecast methods. For this first analysis we use Algorithm I. The DIP is applied to three different point forecast techniques:

1) Holt Winters (HW).

2) The simple linear interpolation (where the linear interpolant is used to compute the prediction at the next time step).

3) The Adaptive Neuro Fuzzy Inference System (ANFIS) with a Gaussian function as mother function. For this purpose we have adopted a toolbox available in the Matlab ${ }^{\circledR}$ programming environment.

Table II shows the performance metrics of the proposed DIP applied to the above listed point forecast methods.

TABLE II

PERFORMANCE OF THE DIP COUPLED WITH THE DIFFERENT POINT FORECAST METHODS.

\begin{tabular}{|c|c|c|c|}
\hline \multicolumn{4}{|c|}{ PICP-PINAW-CWC \% } \\
\hline Time Horizon & $100 \mathrm{~ms}$ & $250 \mathrm{~ms}$ & $500 \mathrm{~ms}$ \\
\hline HW & $95.15-0.059-0.059$ & $95.47-0.12-0.12$ & $95.44-0.27-0.27$ \\
\hline Linear Interpolation & $95.04-0.14-0.14$ & $94.62-0.087-0.19$ & $95.20-0.24-0.24$ \\
\hline \multirow{2}{*}{ ANFIS } & $95.82-0.13-0.13$ & $95.76-0.13-0.13$ & $96.00-0.16-0.16$ \\
\hline
\end{tabular}

By observing Table II it is possible to derive the following conclusions:

1) For a time horizon of $100 \mathrm{~ms}$ the DIP coupled with the $\mathrm{HW}$ is the most performing one in terms of tradeoff between high coverage probability and low interval width. For $250 \mathrm{~ms}$, the $\mathrm{HW}$ and the ANFIS return comparable performance.

2) The ANFIS point forecast method coupled with the DIP is outperforming the other methods for forecast horizons of $500 \mathrm{~ms}$.
3) In general, we have better performance for lower forecast horizons due to the lower variability. The only case where the PICP is lower than the nominal confidence level is the one using linear interpolation at $250 \mathrm{~ms}$ forecast horizon.

\section{Comparison with Benchmark Methods for PI Computation}

In this subsection we compare the proposed DIP with different conventional interval predictors. For this second analysis, we use Algorithm I. We consider the following cases:

1) HW forecast method with the proposed DIP.

2) HW forecast method with PIs based on the variance computation with Gaussian distribution of the error associated with the forecast computation.

3) HW forecast method with PIs based on the basic Bootstrap (BS), [21]. Namely, at each time step we use the empirical quantiles of the error distribution (associated to $\eta$ ) to compute the PIs.

The error distributions of cases 2) and 3) are updated at each sample.

TABLE III

PERFORMANCE COMPARISON OF DIFFERENT PIS COMPUTATION METHODS.

\begin{tabular}{|c|c|c|c|}
\hline \multicolumn{5}{|c|}{ PICP-PINAW-CWC $\%$} \\
\hline Time Horizon & $100 \mathrm{~ms}$ & $250 \mathrm{~ms}$ & $500 \mathrm{~ms}$ \\
\hline DIP & $95.15-0.059-0.059$ & $95.47-0.12-0.12$ & $95.44-0.27-0.27$ \\
\hline GAUSS & $98.30-0.35-0.35$ & $97.60-0.54-0.54$ & $97.71-1.80-1.80$ \\
\hline BS & $92.22-0.12-0.56$ & $90.75-0.15-1.30$ & $92.63-0.27-1.10$ \\
\hline
\end{tabular}

Table III shows that:

1) If we simply look at the CWC value (that combines the other two metrics) we can conclude that, for any time horizon, our DIP coupled with the HW point forecast method is the most performing.

2) The HW coupled with the Gaussian error distribution have high coverage probability but this is counterbalanced by higher values of the PI width.

3) The HW coupled with the Bootstrap has low values of PINAW but PIs are penalized by a coverage probability which is lower than the nominal confidence level.

Fig. 6 gives a more comprehensive evaluation of the PIs obtained with the different interval predictors. Due to the large amount of data, parallel box plots are shown for the comparison. The plotted interval widths are computed such as each distance is normalized with respect to the maximum PV power output. Each box refers to one of the three different interval predictors, considering again the whole time series of Fig. 2 and a time horizon of $250 \mathrm{~ms}$. For each bound predictor the red central mark represents the median, the two blue edges of the box are the 25th and 75th percentiles and 


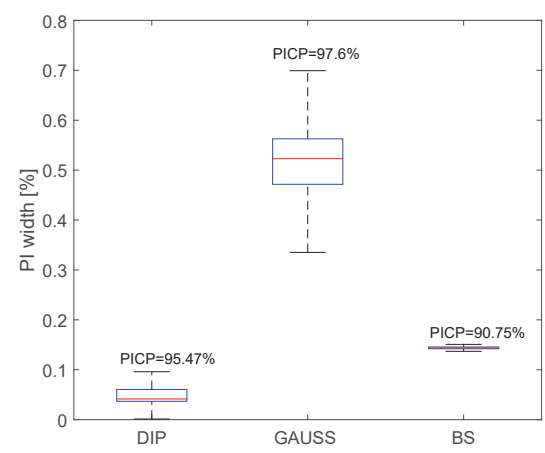

Fig. 6. Box plot of the normalized interval width. 30 days of power data are considered for a time horizon of $250 \mathrm{~ms}$.

the whiskers extend to the maximum and minimum values of the normalized PI width not considered as outliers. For the sake of completeness, the value of the PICP associated to each case is added above the relative box plot. The presented DIP is characterized by the lowest values of the median, quantiles and whiskers but by a higher number of outliers compared to the other two cases (outliers are not plotted here for the sake of clarity). For the DIP, $12 \%$ of the points are outliers, reaching a maximum value of the normalized PI width of $20 \%$. For the Gaussian case, $8 \%$ of the points are outliers with a maximum normalized PI width of $0.96 \%$. For the Bootstrap case, $11 \%$ of the points are outliers with a maximum normalized PI width of $0.41 \%$. The Gaussian method is characterized by a larger interquartile range, which means that the values of the normalized PI are more spread. The PIs are characterized by a higher variability and by more extreme values (represented by the whiskers). However, this is counterbalanced by a high probability coverage. For the BS case the interval widths are less dispersed but, as discussed, we have a poorer coverage probability.

In order to be more conclusive regarding the performance of the DIP, we adjust the value of the nominal confidence level of the other considered methods, aiming at obtaining a comparable value of PICP. Results are shown in Table IV for a time horizon of $250 \mathrm{~ms}$ and exhibit that PINAWs are higher than the one returned by the DIP for similar values of PICP (refer to Table III, $250 \mathrm{~ms}$ ).

TABLE IV

PERFORMANCE OF THE BENCHMARK METODOLOGIES FOR DIFFERENT NOMINAL CONFIDENCE LEVELS $\eta$.

\begin{tabular}{|c|c|c|c|}
\hline$\%$ & $\eta$ & PICP & PINAW \\
\hline GAUSS & 89 & 95 & 0.26 \\
\hline BS & 98 & 95 & 0.36 \\
\hline
\end{tabular}

\section{E. Comparison with Algorithm II}

Table V shows a comparison between algorithms I and II described in Section V. We select the HW as point forecast method and the AC active power value is discretized in 10 equally spaced intervals $(l=10)$. Table $\mathrm{V}$ shows that, for each

TABLE V

Performance COMPARISON BETWEen Algorithm I AND II.

\begin{tabular}{|c|c|c|c|}
\hline \multicolumn{4}{|c|}{ PICP-PINAW-CWC \% } \\
\hline \multirow{2}{*}{ Time Horizon } & $100 \mathrm{~ms}$ & $250 \mathrm{~ms}$ & $500 \mathrm{~ms}$ \\
\hline Alg. I & $95.15-0.059-0.059$ & $95.47-0.12-0.12$ & $95.44-0.27-0.27$ \\
\hline Alg. II & $95.14-0.055-0.055$ & $95.46-0.11-0.11$ & $95.31-0.24-0.24$ \\
\hline
\end{tabular}

time horizon, the addition of a third dimension accounting for the AC active power value allows for improving the performance, in terms of trade-off between PICP and PINAW. From a posteriori analysis, we noticed that performance improves when increasing the number of discretization intervals up to $l=200$, see Table VI. Increasing $l$ leads to a lower PINAW while keeping high value of PICP (higher than $\eta$ ). However, for $l$ higher than 200 we have that PICP decreases below the value of $\eta$, this reducing the quality of the PIs.

The improvement introduced by Algorithm II is particularly evident when encountering high dynamics. This is firstly suggested by Fig. 5 and then confirmed by experimental evidence. As an example, Fig. 7 illustrates the time evolution of the proposed DIP during high PV AC active power fluctuations, for the two algorithms. In both cases the DIP tries to adapt the width of the PI in order to keep the AC power measurements inside the interval. It is possible to see that Algorithm II succeeds and returns PIs that are narrower than those given by Algorithm I.

TABLE VI

PERFORMANCE OF ALGORITHM II FOR TIME HORIZON OF 500 MS AND DIFFERENT DISCRETIZATIONS OF THE AC ACTIVE POWER.

\begin{tabular}{|c|c|c|c|c|}
\hline \multicolumn{5}{|c|}{ PICP-PINAW-CWC \% } \\
\hline$l=5$ & $l=50$ & $l=100$ & $l=200$ & $l=400$ \\
\hline $95.3-0.26-0.26$ & $95.5-0.21-0.21$ & $95.5-0.18-0.18$ & $95.1-0.16-0.16$ & $94.4-0.12-0.27$ \\
\hline
\end{tabular}

\section{F. Deployment into an Industrial Hardware Platform}

In order to discuss the applicability of the proposed DIP, the method is deployed into an industrial microcontroller and operates in the COMMELEC framework in the context of a PV agent. In particular, the DIP has been working with a real-time measurements system since July 2015 as a forecasting tool for the PV plant described in Section IV. The predictor is deployed in a National Instrument CompactRIO 9068, composed by a reconfigurable Artix-7 FPGA, a dual-core ARM Cortex-A9 processor, a 512 MB DDR3 memory and equipped with a NI Linux Real-Time OS. Real-time measurements are acquired by using analog input modules (NI-9215), characterized by an input range of $10 \mathrm{~V}$ and with a maximum sampling frequency 


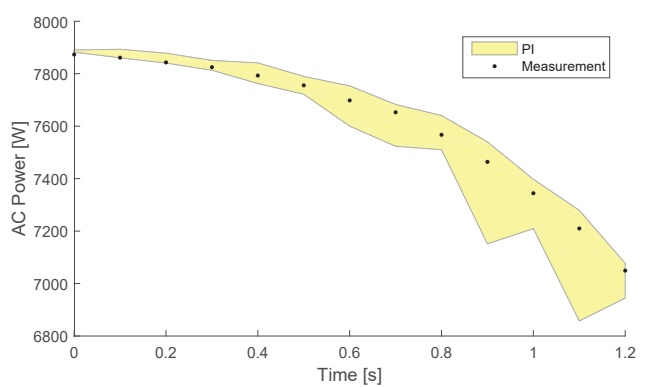

(a) Algorithm I

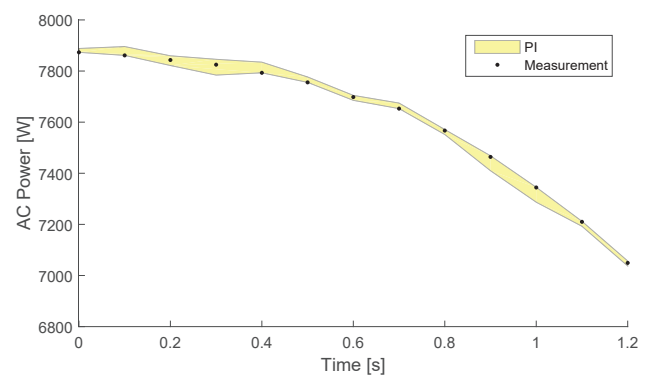

(b) Algorithm II

Fig. 7. PIs (yellow shading) and AC active power measurements (black dots) are plotted for the two algorithms and for high $\mathrm{AC}$ power derivative.

of $100 \mathrm{kHz}$. As described in Section V, the $\mathbf{R}$ matrix is updated at each sample and there is no need to store past data. When a new power measurement is ready, the matrix is updated and it is available for the following PI computations. At each iteration, the whole process including the update of the matrix and the calculation of the PI takes less than $1 \mathrm{~ms}$.

\section{CONCLUSION}

The paper focuses on the computation of sub-second PIs to directly forecast the AC active power output of PV panels equipped with MPPT controllers. The proposed method is based on the experimentally observed correlation between the $\mathrm{AC}$ active power derivative and the error caused by a generic point predictor. An improved approach, accounting for the value of the AC active power as additional explanatory variable, is also presented. The validation dataset consists of an experimental time series of PV power measurements of 30 days. The method does not require any hypothesis on the distribution of the error nor any specific point forecast technique and it is able to account for high $\mathrm{AC}$ active power fluctuations by adapting the PI width.

The proposed DIP is compared with other benchmark methods for PIs (i.e., Gaussian and Bootstrap). Results from this comparison show that the DIP exhibits the best performance in terms of trade-off between high coverage probability and low interval width. A further analysis proves that accounting for the AC active power value, as additional influential variable, allows for improving the performance in particular when encountering high variations. The proposed method has been deployed into an industrial hardware platform and efficiently operates at sub-second update speed for the real-time computation of PIs.

\section{REFERENCES}

[1] D. Torregrossa, J.-Y. Le Boudec, and M. Paolone, "Model-free computation of ultra-short-term prediction intervals of solar irradiance," Solar Energy, 2015.

[2] N. S. D’Souza, L. A. Lopes, and X. Liu, "An intelligent maximum power point tracker using peak current control," in Power Electronics Specialists Conference, 2005. PESC'05. IEEE 36th, p. 172, IEEE, 2005.

[3] E. B. Iversen, J. M. Morales, J. K. Møller, and H. Madsen, "Probabilistic forecasts of solar irradiance using stochastic differential equations," Environmetrics, vol. 25, no. 3, pp. 152-164, 2014.

[4] A. Bernstein, L. Reyes-Chamorro, J.-Y. Le Boudec, and M. Paolone, "A Composable Method for Real-Time Control of Active Distribution Networks with Explicit Power Setpoints. Part I: Framework," Electric Power Systems Research, vol. 125, pp. 254-264, 2015.

[5] L. Reyes-Chamorro, A. Bernstein, J.-Y. Le Boudec, and M. Paolone, "A Composable Method for Real-Ttime Control of Active Distribution Networks with Explicit Power Setpoints. Part II: Implementation and Validation," Electric Power Systems Research, vol. 125, pp. 265-280, 2015.

[6] R. H. Inman, H. T. Pedro, and C. F. Coimbra, "Solar forecasting methods for renewable energy integration," Progress in Energy and Combustion Science, vol. 39, no. 6, pp. 535 - 576, 2013.

[7] H. M. Diagne, P. Lauret, and M. David, "Solar irradiation forecasting: state-of-the-art and proposition for future developments for small-scale insular grids," in WREF 2012-World Renewable Energy Forum.

[8] A. Mellit, H. Eleuch, M. Benghanem, C. Elaoun, and A. M. Pavan, "An adaptive model for predicting of global, direct and diffuse hourly solar irradiance," Energy Conversion and Management, vol. 51, no. 4, pp. 771-782, 2010.

[9] D. Yang, P. Jirutitijaroen, and W. M. Walsh, "Hourly solar irradiance time series forecasting using cloud cover index," Solar Energy, vol. 86, no. 12 , pp. 3531-3543, 2012

[10] C. W. Chow, B. Urquhart, M. Lave, A. Dominguez, J. Kleissl, J. Shields, and B. Washom, "Intra-hour forecasting with a total sky imager at the \{UC\} san diego solar energy testbed," Solar Energy, vol. 85, no. 11, pp. $2881-2893,2011$.

[11] R. Marquez and C. F. Coimbra, "Short term dni forecasting with sky imaging techniques."

[12] H. T. Pedro and C. F. Coimbra, "Assessment of forecasting techniques for solar power production with no exogenous inputs," Solar Energy, vol. 86, no. 7, pp. 2017-2028, 2012.

[13] P. Bacher, H. Madsen, and H. A. Nielsen, "Online short-term solar power forecasting," Solar Energy, vol. 83, no. 10, pp. 1772 - 1783, 2009.

[14] H. T. Pedro and C. F. Coimbra, "Nearest-neighbor methodology for prediction of intra-hour global horizontal and direct normal irradiances," Renewable Energy, vol. 80, pp. 770 - 782, 2015.

[15] Y. Chu, M. Li, H. T. Pedro, and C. F. Coimbra, "Real-time prediction intervals for intra-hour dni forecasts," Renewable Energy, vol. 83, pp. 234-244, 2015.

[16] P. Pinson and G. Kariniotakis, "Conditional prediction intervals of wind power generation," Power Systems, IEEE Transactions on, vol. 25, no. 4, pp. 1845-1856, 2010.

[17] P. Pinson and J. Tastu, "Discussion of prediction intervals for short-term wind farm generation forecasts and combined nonparametric prediction intervals for wind power generation," Sustainable Energy, IEEE Transactions on, vol. 5, no. 3, pp. 1019-1020, 2014.

[18] J.-Y. Le Boudec, Performance Evaluation of Computer and Communication Systems. EPFL Press, Lausanne, Switzerland, 2010.

[19] R. Hyndman, A. B. Koehler, J. K. Ord, and R. D. Snyder, Forecasting with exponential smoothing: the state space approach. Springer Science \& Business Media, 2008.

[20] A. Khosravi, S. Nahavandi, and D. Creighton, "Prediction intervals for short-term wind farm power generation forecasts," Sustainable Energy, IEEE Transactions on, vol. 4, no. 3, pp. 602-610, 2013.

[21] A. C. Davison and D. V. Hinkley, Bootstrap methods and their application, vol. 1. Cambridge university press, 1997. 